\title{
Water Absorption and Degraded Stress Relaxation Behavior in Water-Borne Anticorrosive Urethane/Epoxy Coatings
}

\author{
Yukitoshi Takeshita $^{1 *}$, Ethan Becker ${ }^{2}$, Seizo Sakata ${ }^{1}$, Takuya Kamisho ${ }^{1}$, Takashi Miwa ${ }^{1}$ and Takashi Sawada ${ }^{1}$ \\ 1. NTT Energy and Environment Systems Laboratories, Nippon Telegraph and Telephone Corporation, Tokyo 180-8585, Japan \\ 2. Chemistry and Engineering Physics, University of Wisconsin-Platteville, WI 53818, USA
}

\begin{abstract}
The kinetics of water absorption in water-borne anticorrosive urethane/epoxy coatings, which were actually introduced in the industrial field, are studied. It is found that the high water affinity of a water-borne coating supports a higher saturated water content, $M_{\infty}$, and helps to facilitate absorption $D$. The three parameters of stretched exponential function called the William-Watt equation, $E_{o}, \tau$, and $\beta$, are determined to fit the degraded stress relaxation behavior in the water absorption process because this function quantitatively describes the relaxing ability and has been successfully used by a number of researchers. An increasing in the water content is shown to correlate strongly with a decrease in $E_{o}$ and $\beta$ early in the absorption process between $M_{t} / M_{\infty}=0$ and $M_{t} / M_{\infty} \approx 0.5$. The adhesive characteristics of the coatings are correlated with water content, and shown to exhibit higher cohesive failure in coating epoxies under saturated conditions. This suggests that water interferes with the intermolecular bonding between polymer chains which degrades the bulk materials ability to diffuse stress concentrations and reduces its overall strength.
\end{abstract}

Key words: Water absorption, stress relaxation behavior, water-borne coating, urethane epoxy, maxwell model.

\section{Introduction}

Anticorrosive polymer coatings are widely used to protect steel against corrosion [1]. These coatings are very commonly used on, for example, large steel structures in telecommunication or electric power facilities. The coatings typically have a multi-layer structure, with the bottom layers functioning to bond the coating to the steel substrate, and the top layers providing primary protection and pigmentation. Conventional coating films are typically solvent based and contain a high percentage of VOCs (volatile organic compounds), which are environmentally damaging and pose human health risks. Recent developments have led to the introduction of water-borne low VOC coating systems. Research into the bulk material properties of these low VOC

\footnotetext{
*Corresponding author: Yukitoshi Takeshita, Ph.D., research fields: polymer-based materials, nano-structured coating and corrosion technology. E-mail: takeshita.yukitoshi@lab.ntt.co.jp.
}

coatings is necessary to understand their degradation process.

The viscoelastic properties of water-borne coatings in an artificially accelerated environment or during outdoor exposure have been investigated with the aim of determining degradation process quantitatively [2-6]. Here, we focus attention on water molecules as an aggressive factor, and attempt to determine their contribution to static stress relaxation behavior as a mechanical property of coatings. A number of authors have employed the W-W (Williams-Watts) equation [7], a stretched exponential function, to analyze relaxation behavior. This function well describes the time dependence of a relaxing quantity. This attenuation behavior has been observed for a wide variety of physical quantities in many different systems and research areas. We adopt this function for water-borne urethane/epoxy coatings in the water absorption process, and attempt to indicate the degree of degradation of the relaxing ability while showing 
quantitative parameters through fitting with the W-W equation. In this respect, in past research, it has been difficult to give a definite physical meaning to each parameter. We therefore make an effort to give an interpretation to each of parameters.

In this study we first attempt to determine the water sorption properties of two water-borne, and two solvent-based coating film systems. The water sorption properties of a material can be characterized by two primary properties, the saturated water content and the diffusion coefficient [6]. These two properties are measured for individual layers of each coating film system, multi-layer samples of each coating film, and samples of each coating bonded to a steel substrate. By comparing individual layers, multi layers, and substrate bonded samples we can determine the effects that the bulk material, the interface between separate layers, and the interface between coating and steel have on the overall properties of the coating.

To characterize the contribution of water absorption to the bulk material properties, two tests are performed on dry and saturated samples of each coating. Stress relaxation measures the rate of decay of stress incurred in a coating after being deformed by some applied strain. These data are correlated with the fractional water content of each coating at the time of testing. Finally adhesion testing determines the ability of a coating under tensile stress to remain bonded to a steel substrate in both dry and saturated conditions.

\section{Theory}

\subsection{Water Absorption}

Percent water content, $M_{t}(\%)$ at a given time $t$ can be determined by the Equation:

$$
M_{t}(\%)=\frac{m_{t}-m_{0}}{m_{0}} \times 100
$$

where, $m_{0}$ and $m_{t}$ are the initial dry weight and the weight of the sample at time $t$, respectively. The saturated water content, $M_{\infty}$ can be found by measuring $M_{t}$ as $t$ approaches infinity. As $t$ approaches infinity the mass of the sample will asymptotically approach a stable value, $M_{\infty}$.

If the absorption behavior is expressed by the Fickian equation of diffusion, the water uptake and diffusion process is described by the following Equation at the early absorption stage [8]:

$$
\frac{M_{t}}{M_{\infty}}=\frac{4}{\pi^{1 / 2}}\left(\frac{D t}{l^{2}}\right)^{1 / 2}
$$

where, $D$ is the diffusion coefficient and $l$ is the thickness of the sample. A plot of $M_{t} / M_{\infty}$ as a function of $t^{1 / 2} / l$ can be created, and the initial slope of the graph calculated. By rearranging Eq. (2), the initial slope of the plot can be related to $D$ :

$$
D=\frac{\pi}{16} R^{2}
$$

where, $R$ is the initial slope of the plot. A weighted least squares fit was used to determine the value of $R$, with the weighting factor equal to $1 / \sigma^{2}$, where $\sigma^{2}$ is the variance of the measured values for each point. An example of this fitting method can be seen in Fig. 1.

As Eq. (2) is only valid during the early stages of absorption, the criterion $\frac{M_{t}}{M_{\infty}}<0.6$ was chosen to expressly define the "early stages". Some authors have chosen different values in their analyses of various materials [7-10]. However as there is no commonly accepted value it is necessary to determine one that fits the particular experiment. The chosen criterion falls within the range of values used by other authors, and describes the measured behavior well since the absorption curve of $M_{t} / M_{\infty}$ vs. $t^{1 / 2} / l$ is reasonably linear within the $\frac{M_{t}}{M_{\infty}}<0.6$ range for all measured samples.

\subsection{Stretched Exponential Relaxation}

Stress relaxation behavior can be described for a simple Maxwell model viscoelastic material using an exponential decay function [11]. The Maxwell model represents a viscoelastic material with a dashpot, 


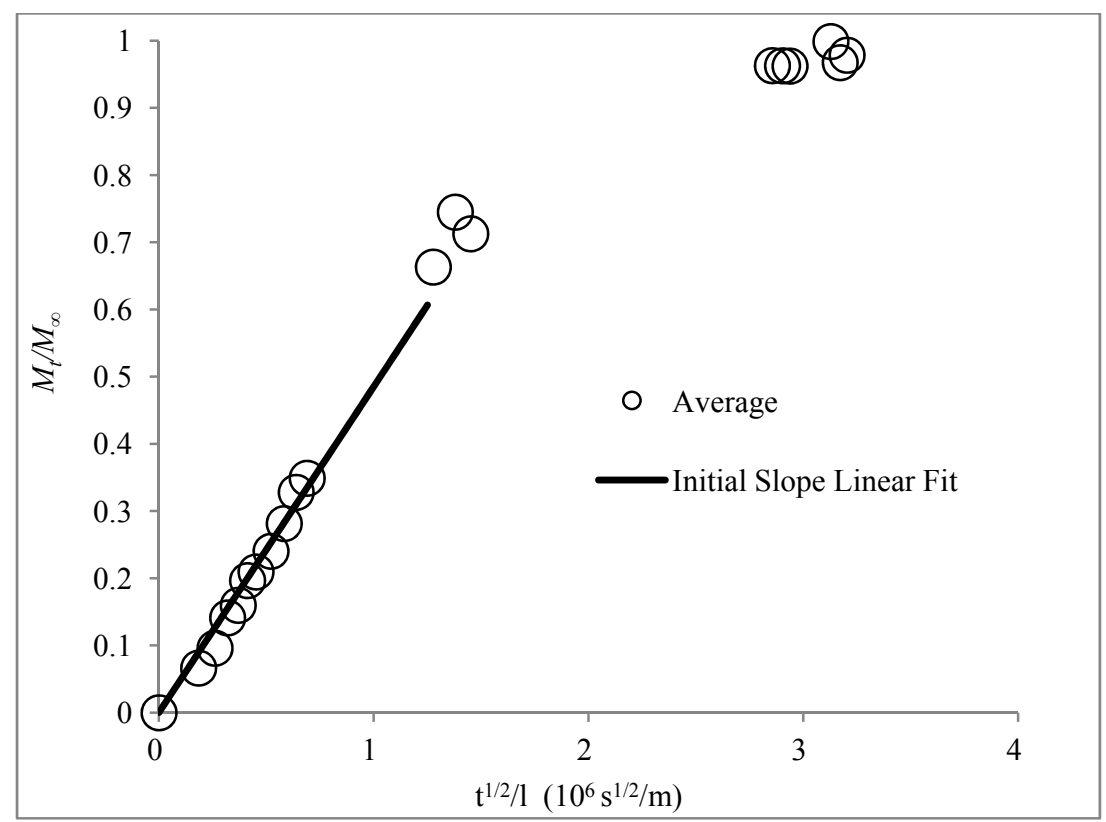

Fig. 1 Plot of $M_{t} / M_{\infty}$ vs. $t^{1 / 2} / l$ used to calculate $D$.

namely the viscous component of the material with a viscosity $\eta$, and a spring, namely the elastic component with an elastic modulus $E$, in series as shown in Fig. 2. If an instantaneous strain is applied to a Maxwell model viscoelastic material the stress will decay exponentially, with a relaxation time $\tau$, as described by Eq. (4).

$$
\sigma(t) / \sigma_{0}=e^{-\left(\frac{E}{\eta} t\right)}=e^{-(t / \tau)}
$$

where, $\sigma(\mathrm{t})$ is the stress in the material at a time $t, \sigma_{0}$ is the stress at $t=0$ after an instantaneous application of strain. $\tau$ is the time constant of the system and is equal to the viscosity divided by the modulus of elasticity. Expansion to a generalized Maxwell model, or Maxwell-Wiechertmodel, which consists of several Maxwell model components with different $E$ and $\eta$ values in parallel, as shown in Fig. 3, produces a function where the relaxation behavior is described as a superposition of multiple exponentially decaying modes.

$$
\sigma(t) / \sigma_{0}=\sum_{p=1}^{p \geq 1} g_{p} e^{-\left(t / \tau_{p}\right)}
$$

where, $\tau_{p}$ is the relaxation time and $g_{p}$ is the normalized intensity of the $p^{\text {th }}$ relaxation mode. The

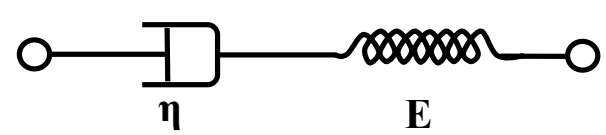

Fig. 2 Diagram of Maxwell model viscoelastic material.

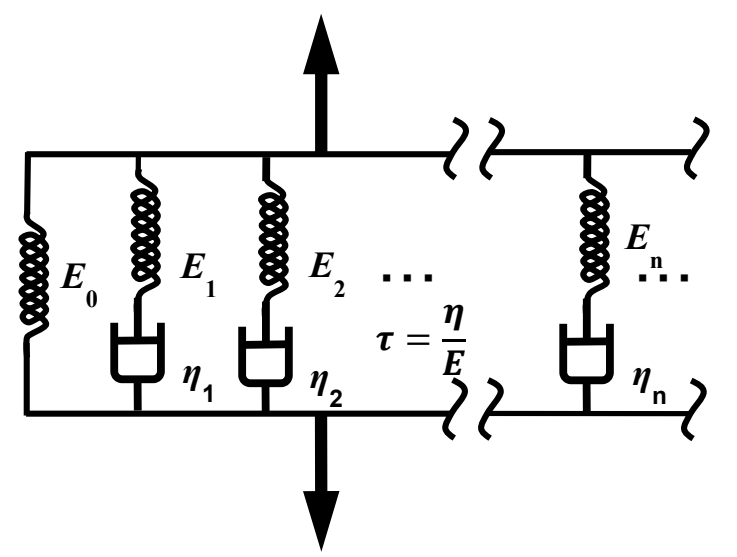

Fig. 3 Diagram of generalized Maxwell model viscoelastic material.

complex nature of the Equation typically results in it being simplified into a stretched exponential function, commonly called the W-W Equation [7].

$$
\varphi(t)=\sigma(t) / \sigma_{0}=e^{-(t / \tau)^{\beta}}
$$

where, the stretching exponent $\beta$, with bounds $0<$ $\beta<1$, represents the relative distribution of the relaxation modes; a decrease in $\beta$ corresponds to an increase in the distribution of relaxation times, $\tau_{p}$, of 
the individual components of the system [12-15]. In this equation $\tau$ represents a "characteristic relaxation time" for the system as a whole. $\varphi(t)$ is the ratio of the stress at a time " $\mathrm{t}$ " to the initial stress.

Eq. (6) assumes an instantaneous application of strain, $\varepsilon^{*}$, on the sample. As this is not possible, strain must be applied over a finite period of time, $t^{*}$, as shown in Fig. 4. This application of strain at a finite rate results in the samples exhibiting a departure from the theoretical behavior for a time $\mathrm{q}^{*}$, where $\mathrm{q}$ is commonly held to be about 10 , after which the observed behavior should match the theoretical behavior within $1 \%$ error [16].

The three parameters of this equation, $\sigma_{0}, \tau$ and $\beta$ are not independent, thus it is necessary to determine one parameter independently before solving for the other two [17]. In this experiment $\sigma_{0}$ was taken to be the maximum stress attained by the sample during testing. $\sigma_{0}$ may be actually higher as some relaxation occurs as the sample is being drawn, however this approximation is close enough to provide an accurate description of the behavior of the sample.

Once an initial value of $\sigma_{0}$ is determined the logarithm of both sides of Eq. (6) can be taken twice to yield.

$$
\ln [-\ln \varphi(t)]=\beta(\ln t-\ln \tau)
$$

A plot of $\ln [-\ln \varphi(t)]$ vs. $\ln t$ yields a straight line with slope $\beta$ and $y$-intercept $-\beta \ln \tau$ [18]. From the calculated values of $\beta$ and $\tau$ a plot of $\sigma(\mathrm{t}) / \sigma_{0}$ vs. t can be made for an instantaneously applied strain as shown in Fig. 5.

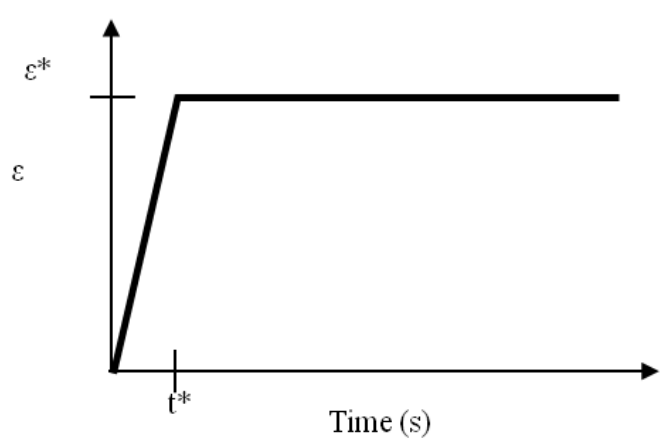

Fig. 4 Application of strain over finite time $t^{*}$.

\section{Experiments}

\subsection{Materials}

We used two types of base substrates, an SS400 steel plate $(50 \times 100 \times 3.2 \mathrm{~mm})$ that was galvanized and sweep-blasted and a PTFE (polytetrafluoroethylene) plate. The former was for the coating sample on the substrate, and the latter was for the free coating sample in which the coating had been peeled off from the PTFE plate. The substrate of the steel or PTFE is cleaned and consecutively painted with each of the three layers of the coating. A drying time of $24 \mathrm{~h}$ is allowed between the paintings of each layer. After the applied layer(s) have dried, the substrate is allowed to rest for at least 30 days until it reaches an age appropriate for testing.

Four different coatings (A, B, C and D) were used in this study. A and B are water-borne coatings comprised of an epoxy bottom and middle layer and a urethane top layer with thicknesses of 50,50 and $25 \mu \mathrm{m}$. The urethane layers of coatings A and B are the same, while the epoxy layers of coating $A$ have a lower epoxy equivalent ratio than coating $\mathrm{B}$ in which the ratio of the epoxy equivalent to the amine active hydrogen equivalent is lower, suggesting that $\mathrm{A}$ has a relatively high number of unreacted epoxide groups. Coatings $\mathrm{C}$ and $\mathrm{D}$ are solvent based coatings, which also consist of an epoxy bottom and middle layer and a urethane top layer with thicknesses of 50, 50 and $25 \mu \mathrm{m}$. $\mathrm{C}$ and $\mathrm{D}$ are strong and weak dissolving solvent types, respectively, in which the amount of VOCs of $\mathrm{C}$ is generally greater than that of $\mathrm{D}$.

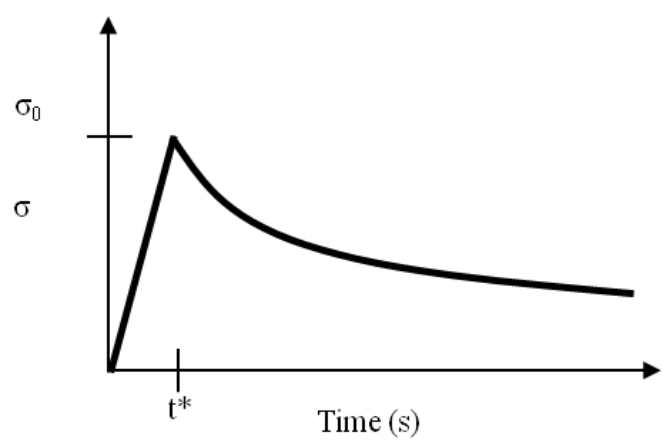




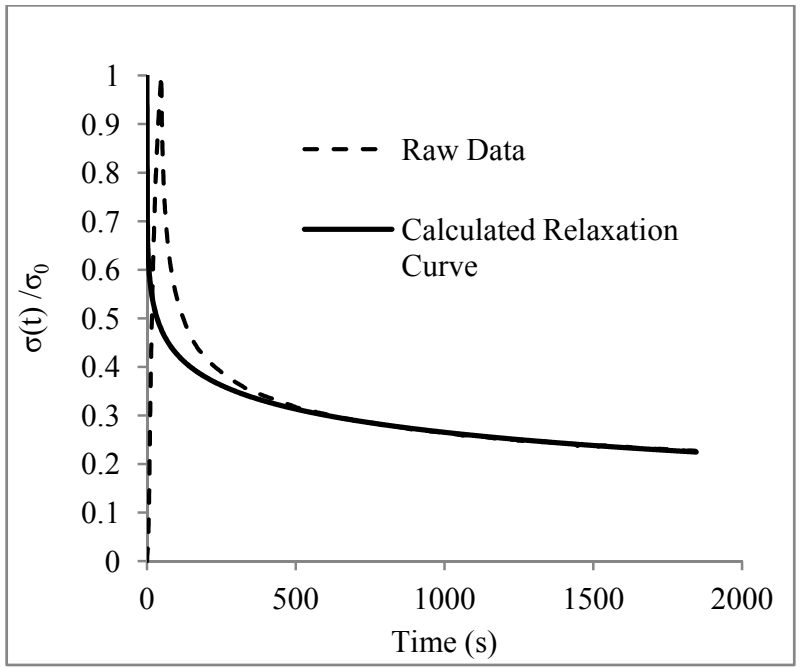

Fig. 5 Example calculated relaxation curve for an instantaneously applied strain, fitted to raw data.

\subsection{Water Absorption}

Whole coating and individual layer samples were prepared by cutting the film on the PTFE substrate into $20 \mathrm{~mm} \times 20 \mathrm{~mm}$ squares, and peeling each square from the substrate. The samples were cut sufficiently far from the edge of the substrate so that the entire sample was roughly uniform. Whole coating samples bonded to steel substrates were tested without removing the sample from the substrate or cutting the sample into smaller sections.

To measure the absorption behavior, each prepared sample was initially weighed and its thickness measured with a micrometer. The thickness was measured at five points on the sample, namely at each corner and at the center of the sample, with the mean of these five measurements taken to reflect the actual thickness of the sample. The samples were then immersed in a bath of distilled water. At regular intervals each sample was removed from the water, wiped dry with a paper towel, and weighed before being immediately returned to the water bath. This process was repeated until the samples reached a constant mass.

For samples bonded to a steel substrate the mass of the steel must be subtracted from the measured mass, to obtain the mass of the sample. To calculate the mass of the sample, the area density of a sample of the same coating that has been removed from its PTFE substrate was calculated. The surface area of the coating on the steel substrate was measured and multiplied by the calculated area density to determine the original mass of the coating and the steel substrate. The percent mass absorbed could then be calculated using the following Equation.

$$
M_{t}(\%)=\frac{\left(m_{t}-m_{s}\right)-m_{c}}{m_{c}}
$$

where, $m_{c}$ is the original dry mass of the coating, $m_{s}$ is the mass of the steel, and $m_{t}$ is the mass of the coating + steel + absorbed water at time $t$. The sorption of galvanized substrate was ignored in this paper.

\subsection{Stress Relaxation}

Samples were prepared by removing sheets of whole coating films from a PTFE substrate and stamping out dumbbell shape ISO 37:2005 [19] test samples. The dumbbell shapes have a neck that is $2 \mathrm{~mm}$ wide by $10 \mathrm{~mm}$ long. The samples were numbered, weighed and the thickness was measured in three places along the neck of the sample using a micrometer.

Tested samples with some fractional content of absorbed water were immersed in distilled water long enough to reach an absorbed water content of $M_{t} / M_{\infty}=$ 0.5 (approx. $16 \mathrm{~h}$ ), or $M_{t} / M_{\infty}=1$ (approx. 5 days). During testing the mass of the sample was measured before and after the test to determine $M_{t}(\%)$ both immediately before and immediately after the test, and the mean of these two values representing the $M_{t}(\%)$ value was used during analysis.

All measurements were carried out on a tensile testing machine, ToyoseikiStrograph VG 10-E. Samples were mounted between two testing clamps and a small amount of tension was applied to the samples as they were being loaded into the apparatus to ensure that there was no slack in the sample.

All the samples were drawn with the same strain rate of $0.5 \mathrm{~mm} / \mathrm{min}$. The maximum applied strain of each coating differed. One dry sample of each coating was drawn at $0.5 \mathrm{~mm} / \mathrm{min}$ until failure to determine its 
maximum stress/strain. The point of failure was measured and the strain used for testing was chosen to be approximately $75 \%$ of the maximum strain. The ambient temperature and humidity ranged from 18.9-22.0 ${ }^{\circ} \mathrm{C}$ and $18 \%-52 \%$ RH during the testing period.

The testing time for each sample was chosen to be at least 20 times the time necessary to draw the sample. This ensured that there was sufficient data beyond time $\mathrm{qt}^{*}$ which significant data could be analyzed.

\subsection{Adhesive Strength}

Adhesive strength measures the ability of a coating to bond to a steel substrate. Measurements determine the maximum tensile stress that can occur in the coating before failure and the primary plane of failure.

Saturated samples were first allowed to absorb water for sufficient time to reach $M_{t} / M_{\infty} \approx 1$. Dry and saturated samples were prepared by attaching a $20 \mathrm{~mm}$ diameter dolly with an adhesive epoxy to the surface of the sample. The adhesive epoxy was allowed to cure for $24 \mathrm{~h}$ before being tested.

All measurements were carried out on a tensile testing machine. The coating and steel substrate were held down with 4 clamps while a testing arm was clamped to the dolly that had been bonded to the surface of the sample. The applied force at the point of failure was recorded along with the location of the failure plane. Before testing the area immediately around the dolly was cut to minimize the effects of shear stress during testing.

\section{Results and Discussion}

\subsection{Saturated Water Content}

Fig. 6 shows the absorption curves for all coatings freely immersed in water, and bonded to a steel

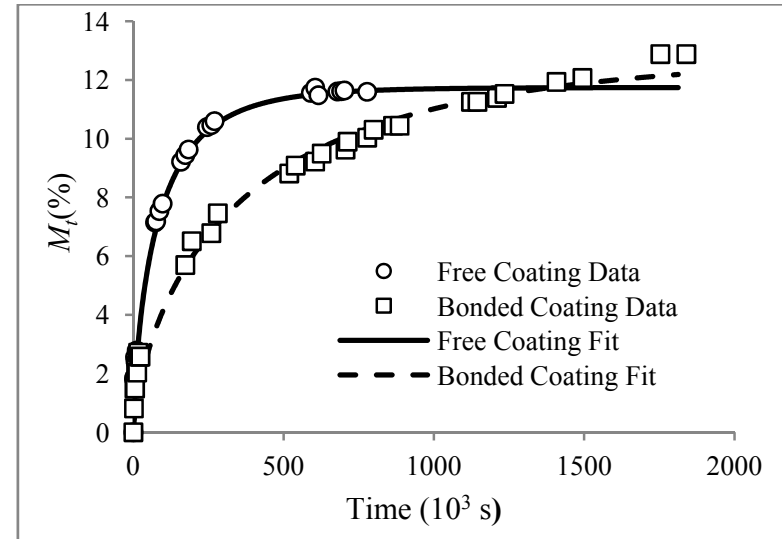

(a)

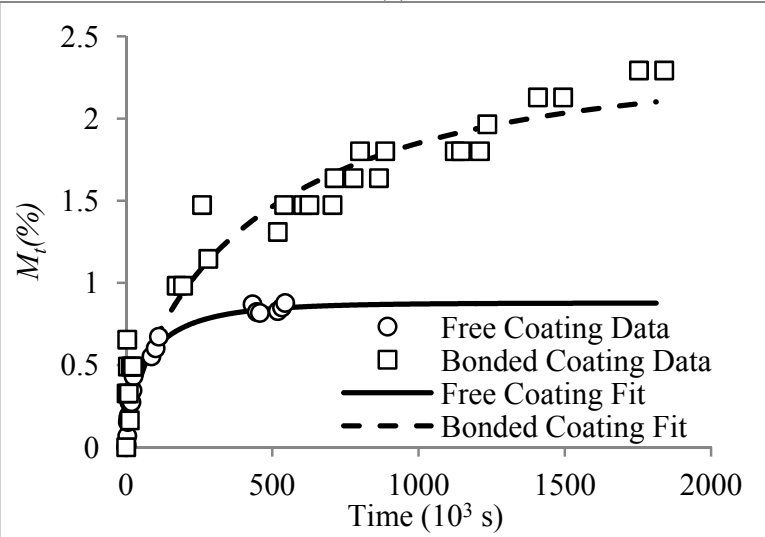

(c)

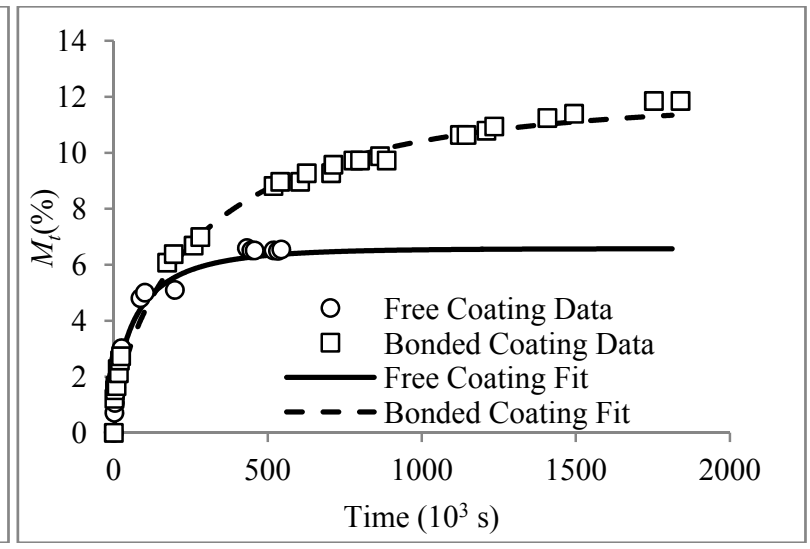

(b)

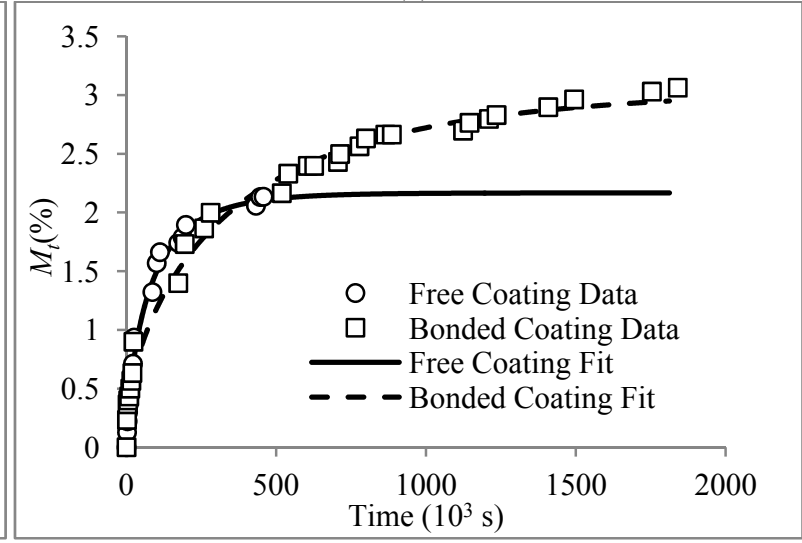

(d)

Fig. 6 Absorption curve of free and steel bonded coatings. Results for coatings A, B, C and D are shown in (a), (b), (c) and (d), respectively. 
substrate. The saturated water content for all the multi-layer coatings, $M_{\infty}(\%)$, shown in Table 1 , was measured for coatings that were 70 days old. Coatings A and B had significantly higher values of $11.6 \%$ and $6.9 \%$, respectively, than coatings $\mathrm{C}$ and $\mathrm{D}$, which were $0.8 \%$ and $2.1 \%$, respectively.

The higher $M_{\infty}(\%)$ values obtained for coatings A and $\mathrm{B}$ are due to them being water based whereas coatings $\mathrm{C}$ and $\mathrm{D}$ are solvent based. This causes coatings A and B to have a higher affinity to water. Additionally, the comparatively low epoxy equivalent ratio of the epoxy layer of coating A relative to coating B means that there are far more unreacted epoxy functional groups, which provide additional bonding sites for water through dipole interactions. The top (urethane) layer of coatings A and B is the same and thus should not contribute to any differences between the $M_{\infty}(\%)$ values of the two coatings. The low $M_{\infty}(\%)$ in coating $\mathrm{C}$ compared with coating $\mathrm{D}$ can most likely be attributed to the former being a strong solvent type coating, whereas the latter is a weak solvent type coating.

Fig. 6 also compares free coatings (70 days old) with coatings bonded to a steel substrate (40 days old). The bonded coatings all exhibited a higher saturated water content than isolated coatings. $12.8 \%, 11.8 \%$, $2.3 \%$ and $3.1 \%$ for coatings $\mathrm{A}, \mathrm{B}, \mathrm{C}$, and $\mathrm{D}$, respectively, represent an approximately $1.0 \%-4.9 \%$ increase in saturated water content ([II]-[I] in Table 1). This indicates that a certain amount of water is stored in the interface between the bottom layer and the steel substrate for each of these coatings. This significant increase suggests that there is a factor influencing the storage of water between the coating and the substrate.

To compare the properties of each layer of the coating individually with the properties of the multi-layer coating, the $M_{\infty}(\%)$ values were also measured for the individual layers of each coating from samples that were 75 days old as shown in Table 2 . The $M_{\infty}(\%)$ values of the whole coating film can be estimated from the weighted average $M_{\infty}(\%)$ value for each layer of the film, with the weighting factor being the thickness of the layer in the whole film. $M_{\infty}(\%)$ values calculated in this manner can allow us to determine the effect of water stored in the interface between layers.

For coatings $\mathrm{A}$ and $\mathrm{B}$, the differences between the weighted average and the measured values ([II]-[I]) in Table 2 were $3.7 \%$ and $1.0 \%$, respectively, indicating that there is water stored between the layers of the coating. For coatings $\mathrm{C}$ and $\mathrm{D}$ the weighted average and measured values differed by about only $0.1 \%$, indicating that there was little to no water stored between the layers.

Table 1 Measured for all coatings.

\begin{tabular}{llll}
\hline & $\begin{array}{l}\text { Whole coating } \\
(\%)[\mathrm{I}]\end{array}$ & Whole coating & $\begin{array}{l}{[\mathrm{II}]-[\mathrm{I}]} \\
(\%)\end{array}$ \\
\hline $\mathrm{A}$ & 11.6 & bonded to steel $(\%)[\mathrm{II}]$ & +1.2 \\
$\mathrm{~B}$ & 6.9 & 12.8 & +4.9 \\
$\mathrm{C}$ & 0.8 & 11.8 & +1.5 \\
$\mathrm{D}$ & 2.1 & 2.3 & +1.0 \\
\hline
\end{tabular}

Table 2 Comparison of measure $M_{\infty}$ value of whole coating with weighted average of individual layers.

\begin{tabular}{llllll}
\hline & Top layer $(\%)$ & $\begin{array}{l}\text { Bottom/middle layer } \\
(\%)\end{array}$ & $\begin{array}{l}\text { Weighted average } \\
(\%)[\mathrm{I}]\end{array}$ & $\begin{array}{l}\text { Whole coating } \\
(\%)[\mathrm{II}]\end{array}$ & $\begin{array}{l}{[\mathrm{II}]-[\mathrm{I}]} \\
(\%)\end{array}$ \\
\hline $\mathrm{A}$ & 5.5 & 8.5 & 7.9 & 11.6 & +3.7 \\
$\mathrm{~B}$ & 5.7 & 5.9 & 5.9 & 6.9 & +1.0 \\
$\mathrm{C}$ & 0.7 & 0.8 & 0.7 & 0.8 & +0.1 \\
$\mathrm{D}$ & 4.0 & 1.5 & 2.0 & 2.1 & +0.1 \\
\hline
\end{tabular}

Weighted average calculated from $M_{\infty}(\%)$ measurements for individual layers. Weighting value equal to thickness of each layer in multi-layer coating film. 
The measured $M_{\infty}(\%)$ values for the individual layers of the bottom/middle exhibit a positive correlation to the coating's water affinity, $\mathrm{A}>\mathrm{B}>\mathrm{D}>$ $\mathrm{C}$, in the same way as the multi-layer coatings. Additionally the amount of water stored in the interface between the individual layers exhibits a positive correlation with the water affinity.

\subsection{Water Uptake Process}

To measure the diffusion coefficient of each coating, the normalized sorption curves are plotted as a function of $t^{1 / 2} / l$ as shown in Fig. 7. The initial stage, in which $M_{t} / M_{\infty}$ is less than 0.6 , appears to present a straight line. A number of other researchers using the same epoxy and urethane samples with a variety of resins and hardeners have successfully used the approach on the assumption that behavior would be treated as Fickian diffusion [20]. Therefore, we believe it to be quite acceptable to consider using a similar approach that follows Fickian diffusion when comparing coatings. The diffusion coefficients, for all the coatings, were calculated using a previously described method and shown in Table 3.

From Table 3 , the $D$ values for the whole coating are ranked from high to low as $\mathrm{A}>\mathrm{B}>\mathrm{D}>\mathrm{C}$. $\mathrm{A}$ strong positive correlation exists between $D$ and $M_{\infty}(\%)$. As several other studies have concluded, this can be attributed to a higher water affinity, which attracts water molecules during absorption [10, 21-24]. This attraction can cause swelling within the cavities of the polymer network, which further increases $M_{\infty}(\%)$ and $D$.

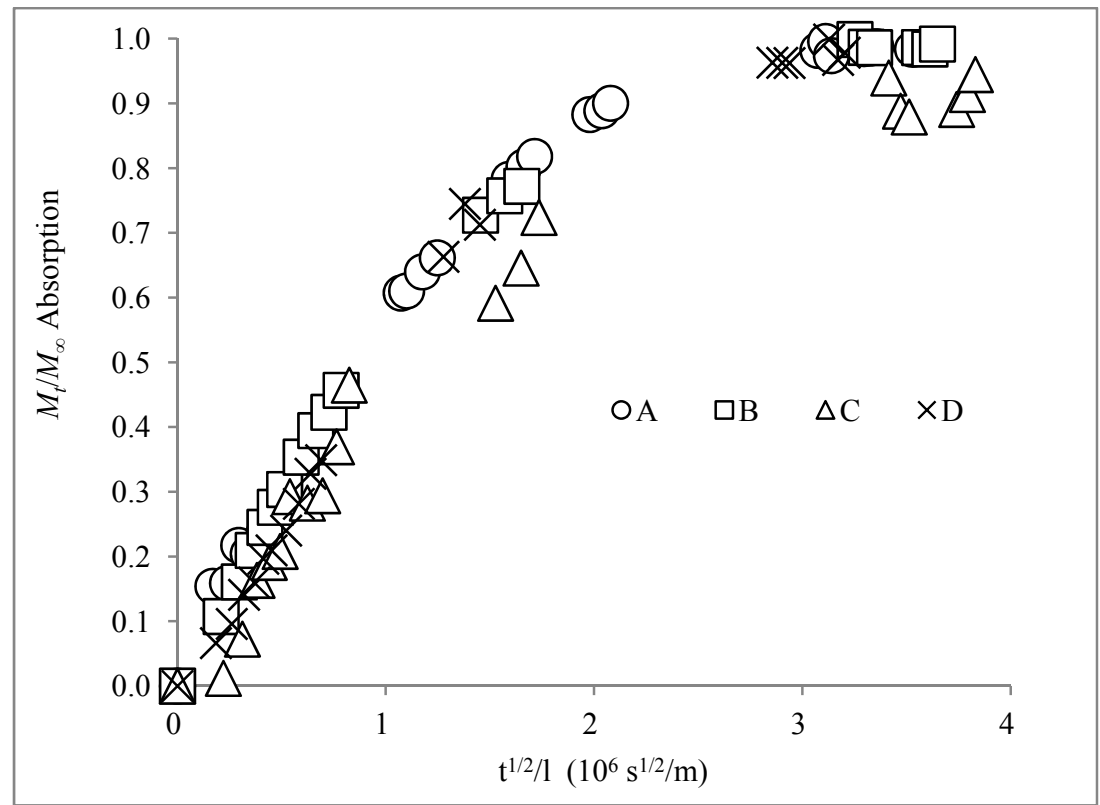

Fig. 7 Normalized water uptake, $M_{t} / M_{\infty}$ absorbed, vs. $t^{1 / 2} / l$ for all coatings.

Table 3 Calculated $D$ values for all coatings.

\begin{tabular}{llllll}
\hline & $\begin{array}{l}\text { Whole coating } \\
\left(\mathrm{m}^{2} / \mathrm{s}\right)\end{array}$ & $\begin{array}{l}\text { Top layer } \\
\left(\mathrm{m}^{2} / \mathrm{s}\right)\end{array}$ & $\begin{array}{l}\text { Bottom/middle layer } \\
\left(\mathrm{m}^{2} / \mathrm{s}\right)\end{array}$ & $\begin{array}{l}\text { Weighted average } \\
\left(\mathrm{m}^{2} / \mathrm{s}\right)\end{array}$ & $\begin{array}{l}\text { Whole coating on steel } \\
\left(\mathrm{m}^{2} / \mathrm{s}\right)\end{array}$ \\
\hline $\mathrm{A}$ & $7.53 \mathrm{E}-14$ & $1.86 \mathrm{E}-13^{*}$ & $3.71 \mathrm{E}-14$ & $6.69 \mathrm{E}-14$ & $6.15 \mathrm{E}-14$ \\
$\mathrm{~B}$ & $6.68 \mathrm{E}-14$ & $1.82 \mathrm{E}-13^{*}$ & $3.80 \mathrm{E}-14$ & $6.68 \mathrm{E}-14$ & $4.60 \mathrm{E}-14$ \\
$\mathrm{C}$ & $3.41 \mathrm{E}-14$ & $9.07 \mathrm{E}-14 *$ & $2.37 \mathrm{E}-15$ & $2.32 \mathrm{E}-14$ & $2.93 \mathrm{E}-14$ \\
$\mathrm{D}$ & $4.62 \mathrm{E}-14$ & $2.38 \mathrm{E}-15$ & $5.68 \mathrm{E}-14$ & $4.60 \mathrm{E}-14$ & $7.20 \mathrm{E}-14$ \\
\hline
\end{tabular}

* Value represents lower bound of $D$.

Weighted average calculated from $D$ measurements for individual layers. Weighting value equal to thickness of each layer in multi-layer coating film. 
The diffusion coefficients of individual layers were also compared with those of multi-layer coatings as shown in Table 3. For all the coatings the diffusion coefficient during absorption for multi-layer films is close to the weighted average of the diffusion coefficients for the individual layers.

A comparison of the $D$ value of the whole coating and that on steel for A, B, and C reveals a relationship where whole coating $>$ coating on steel. A small decrease in $D$ was observed for samples bonded to a steel substrate vs. a free coating. This difference is thought to be caused by the interface between the top and bottom/middle layer. In the free sample, it is not necessary for all the absorbed water to cross this interface, as it can permeate into the film directly through the bottom/middle layer, where as the in the steel bonded sample all water absorbed into the bottom/middle layer must cross this interface.

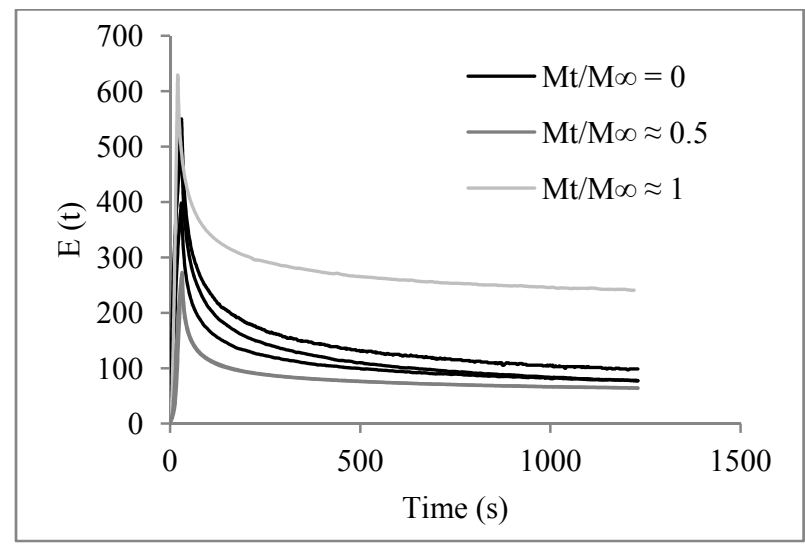

(a)

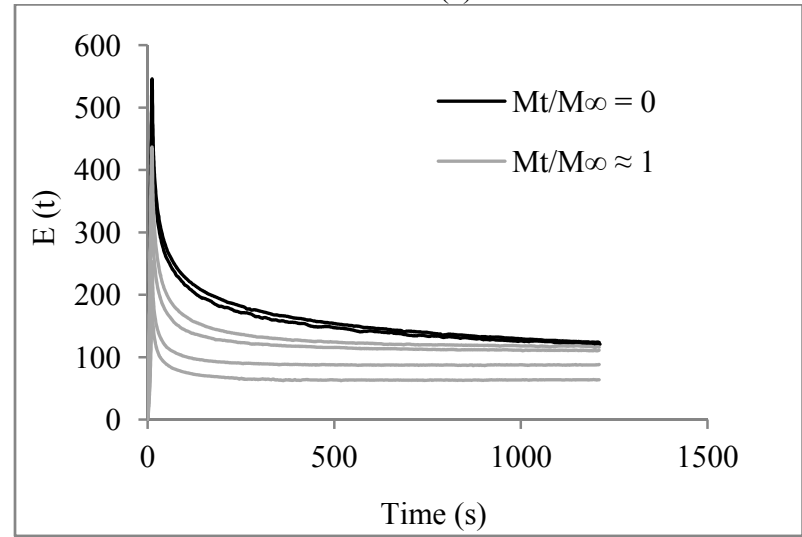

(c)
Look at Fig. 6 again, due to the increased $M_{\infty}(\%)$ and decreased $D$ for samples bonded to a steel substrate relative to the free samples, the absorption curves cross at a certain point as seen in Fig. 6. This crossover point is thought to indicate the time at which water begins to infiltrate the interface between the coating and the steel substrate [25].

\subsection{Stretched Exponential Relaxation Behavior}

Stress relaxation curves for all the coatings are shown in Fig. 8. Note that because the mean values of the $M_{t}$ measurements made before and after testing are used in the analysis when some desorption occurred during the testing of saturated and partially saturated samples, even data points representing the saturated samples have an $M_{t} / M_{\infty}$ value slightly below 1 .

The three parameters of the stretched exponential function, namely $E_{o}, \tau$, and $\beta$ are determined to fit the

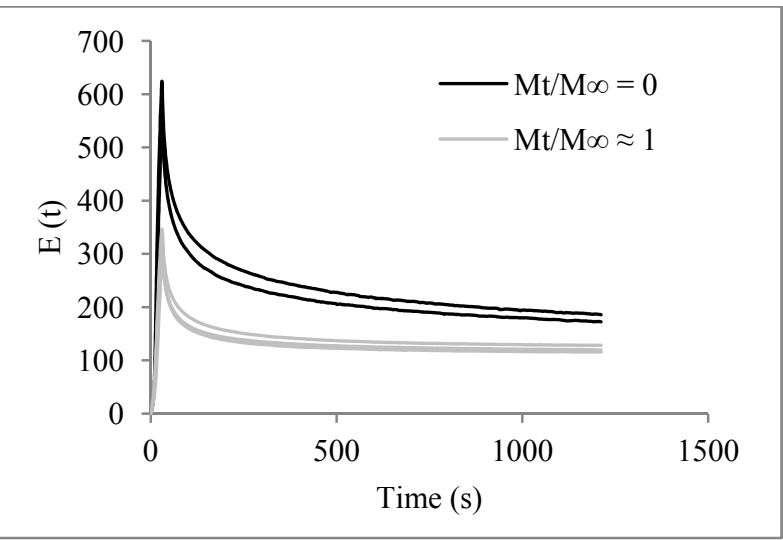

(b)

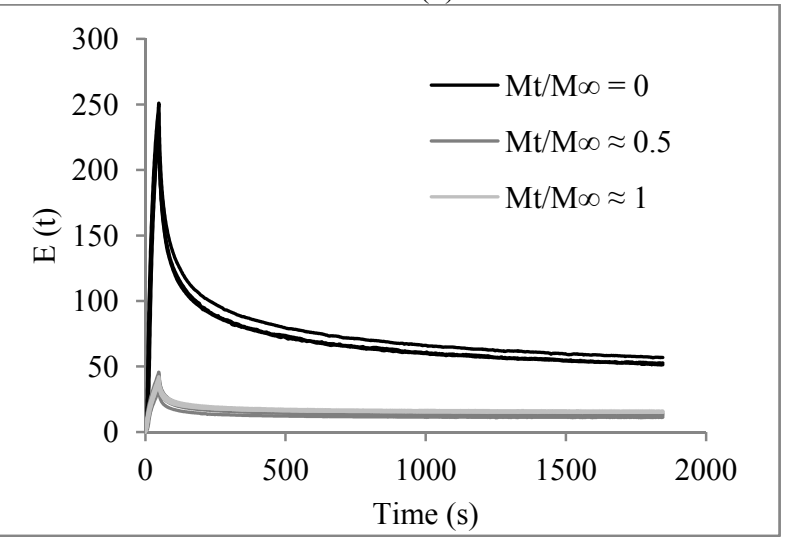

(d)

Fig. 8 Measured stress relaxation curves for all tested samples. Results for coatings A, B, C and D are shown in (a), (b), (c), and (d), respectively. 

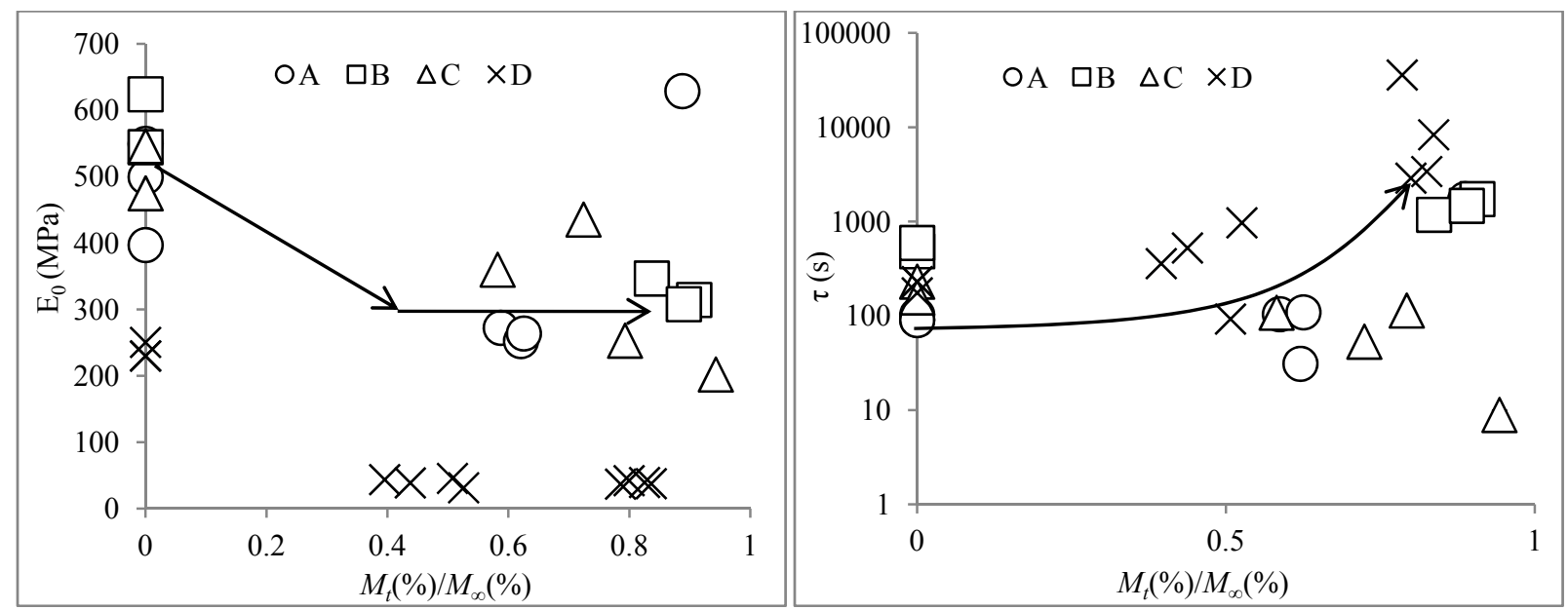

(a)

(b)

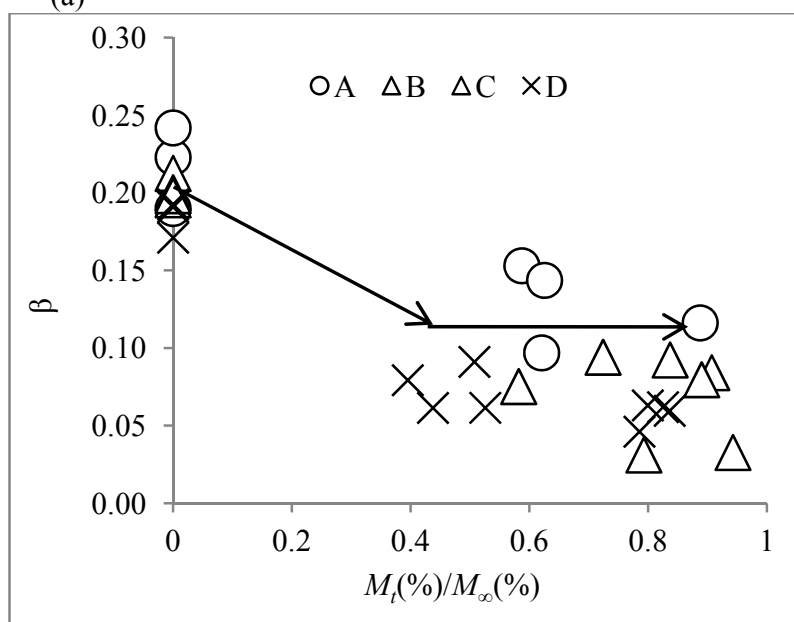

(c)

Fig. 9 Three parameters determined in the W-W equation as a function of $M_{t} / M_{\infty}$ (a) $E_{0}$ vs $M_{t} / M_{\infty}$, (b) $\tau$ vs. $M_{t} / M_{\infty}$, (c) $\beta$ vs. $M_{t} / M_{\infty}$.

stress relaxation behavior in the way described previously and plotted as a function of $M_{t} / M_{\infty}$ in Fig. 9. $E_{o}$ and $\beta$ quickly decreased in the early stages between $M_{t} / M_{\infty}=0$ and $M_{t} / M_{\infty} \approx 0.5$ and leveled off later between $M_{t} / M_{\infty} \approx 0.5$ and $M_{t} / M_{\infty} \approx 1$, as shown in Figs. 9a and 9c. $\tau$ exhibited a roughly exponential increase between $M_{t} / M_{\infty}=0$ and $M_{t} / M_{\infty} \approx 1$, as shown in Fig. 9b.

Water is commonly considered to act as a plasticizing agent in polymers [9, 21, 26-28], which caused a decrease in $E_{0}$ in all the samples as $M_{t} / M_{\infty}$ approached 1. Coatings B, C and D experienced a decrease in $E_{0}$ of $45 \%, 39 \%$ and $84 \%$ respectively between $M_{t} / M_{\infty}=$ 0 and $M_{t} / M_{\infty} \approx 1$. Coating A experienced a decrease of $44 \%$ between $M_{t} / M_{\infty}=0$ and $M_{t} / M_{\infty} \approx 0.5$.
An increase in $\tau$ can be seen for coatings A, B, and $\mathrm{D}$ in Fig. 9b. Coating B experienced an approximately twofold increase while coatings $A$ and $D$ experienced an increase of between 1 and 2 orders of magnitude between $M_{t} / M_{\infty}=0$ and $M_{t} / M_{\infty} \approx 1$. Coatings $\mathrm{A}$ and D experienced an increase of $120 \%$ and $200 \%$, respectively, at $M_{t} / M_{\infty} \approx 0.5$. Coating $\mathrm{C}$ saw a decrease in $\tau$ as $M_{t} / M_{\infty}$ increased, with an approximately $63 \%$ decrease at $M_{t} / M_{\infty} \approx 1$.

All samples exhibited a decrease in $\beta$, with a total decrease between $M_{t} / M_{\infty}=0$ and $M_{t} / M_{\infty} \approx 1$ of $47 \%$, $58 \%, 70 \%$, and $69 \%$ for coatings $\mathrm{A}, \mathrm{B}, \mathrm{C}$, and D, respectively. Coatings $\mathrm{A}$ and $\mathrm{D}$ experienced decreases of $32 \%$ and $60 \%$ at $M_{t} / M_{\infty} \approx 0.5$ as seen in Fig. 9c.

Molecular interactions between polymer chains, 
such as hydrogen and dipole-dipole bonding, cause some degree of reduction in chain mobility [29]. This has the effect of "averaging" or decreasing the distribution of the relaxation times for the individual components of the system. The introduction of water into the polymer network interrupts the intermolecular bonding $[21,22,30]$, which reduces this averaging effect and results in the reduction in $\beta$ for the system as a whole.

The decrease in both $E_{o}$ and $\beta$ between $M_{t} / M_{\infty}=0$ and $M_{t} / M_{\infty} \approx 0.5$ followed by a flattening between $M_{t} / M_{\infty} \approx 0.5$ and $M_{t} / M_{\infty} \approx 1$ is likely caused by the nature of water absorption in polymers. Numerous authors have suggested that water is first absorbed into polymers by bonding with the polar bonding sites on the polymer chains, before filling the free space in the polymer network as unbonded water [22, 31]. Water that is bonded to polymer chains interrupts molecular interactions between polymer chains, which contribute to changes in the bulk material properties. Free water, on the other hand, has little effect on polymer chains, and little influence on the material properties [10, 22]. Our group has reported that most absorbed water molecules are strongly bound to polymer chains, which contributes significantly to a degradation in mechanical strength, followed by weak 1 , weak 2 , and free state of water by means of DSC (differential scanning calorimetry) and attenuated total reflectance Fourier transform IR spectroscopy (ATR FTIR) [32].

Absorption of this nature explains the large change in properties between $M_{t} / M_{\infty}=0$ and $M_{t} / M_{\infty} \approx 0.5$, as well as the relatively small changes between $M_{t} / M_{\infty} \approx$ 0.5 and $M_{t} / M_{\infty} \approx 1$. This conclusion explains both $E_{o}$ and $\beta$ through the same mechanism, and it is supported by a strong positive correlation between $E_{o}$ and $\beta$ as shown in Fig. 10. Values of $E_{o}$ and $\beta$ are normalized against their average value at $M_{t} / M_{\infty}=0$ in Fig. 10 for ease of analysis.

Relaxation time provides a basic description of the relaxation nature of a polymer as it provides the best comparison of the relative amount of time it takes for the initial stress to decay as well as being the most intuitive parameter of the $\mathrm{W}-\mathrm{W}$ equation. $\tau$ describes the point at which the stress in the sample is equal to $1 / e$ its initial value. The complex nature of the $\mathrm{W}-\mathrm{W}$ equation means that all other multiples of $\tau$ have a less significant meaning as the proportion of the initial stress at those points will also depend on $\beta$ (Fig. 11a).

The physical effect of $\beta$ is more difficult to understand than either $E_{0}$ or $\tau$, and can be most aptly characterized as describing the general shape of the relaxation function, with lower values causing a sharper drop early in the relaxation and a flattening of the curve later in the relaxation. Relaxation curves with the same $\beta$ value initially exhibit different relaxation behaviors, but gradually adopt a similar shape. These effects are illustrated in Fig. 11a and $11 \mathrm{~b}$.

Johnston showed [13] that in the W-W equation, Eq. (6), $\tau$ is most indicative of the median value, $\tau_{\text {med }}$, of the distribution of relaxation times, $\tau_{p}$, for the components of the system in Eq. (6), within the range $0.5<\beta<1$. He also showed that for any value of $\beta<1$, $\tau$ is greater than the peak (or most common) value, $\tau_{\text {peak }}$, in the distribution of $\tau_{p}$ and greater than the average value, $\tau_{\text {avg }}$, of $\tau_{p}$. The relationships $\tau_{\text {peak }}<\tau$ and $\tau_{\text {avg }}<\tau$ are caused by an asymmetrical distribution of $\tau_{p}$ for $\beta<1$. Thus, although the $\tau_{\text {peak }}$ and/or $\tau_{\text {avg }}$ may remain the same during the absorption process, a reduction of $\beta$ will lead to a corresponding increase in $\tau$. This can be shown by correlating $\beta$ and $\tau$ in Fig. 12 in which y-axis is shown on a logarithmic scale where a decrease in $\beta$ corresponds to an approximately exponential increase in $\tau$. This relation between $\beta$ and $\tau$ is likely the most significant factor affecting changes in $\tau$ during the absorption process.

It is important to note that the distribution of $\tau$, created by the W-W equation does not necessarily precisely match with the actual distribution of the relaxation times $\tau_{p}$. Conclusions drawn about the nature of the relationship between $\tau$ and $\beta$ are properties 


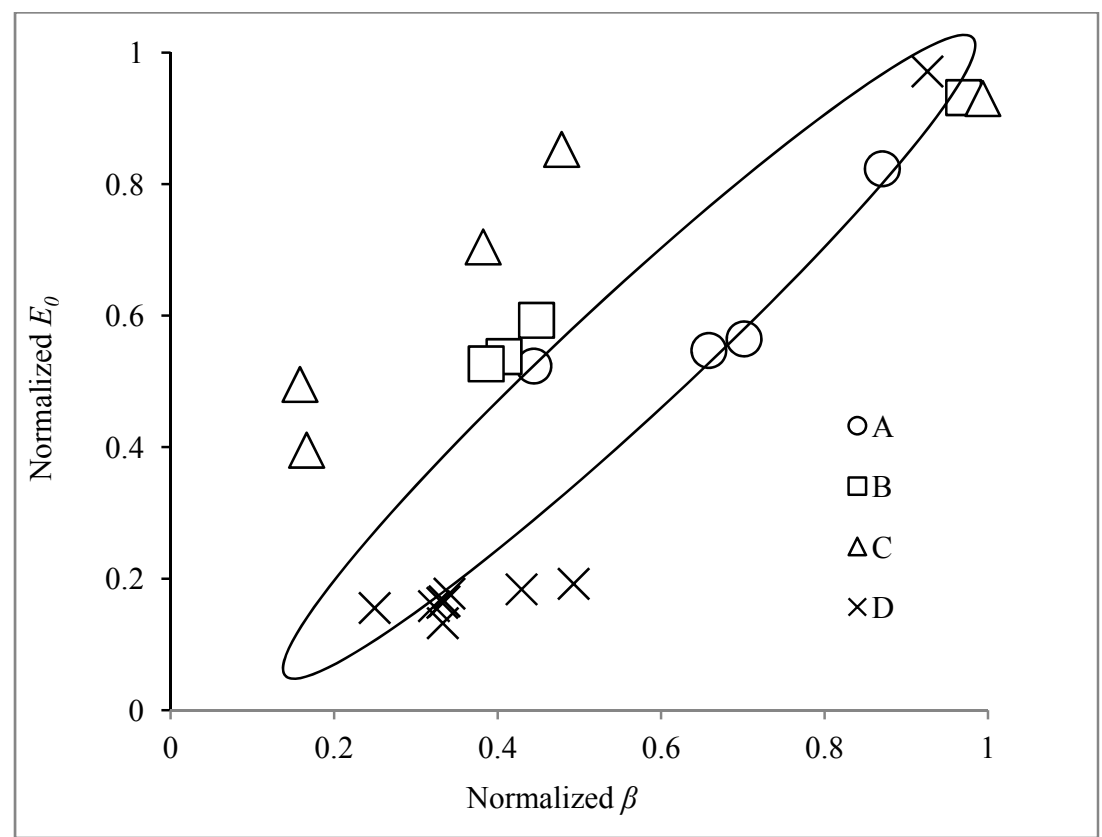

Fig. 10 Correlation between normalized $E_{0}$ and normalized $\beta$.

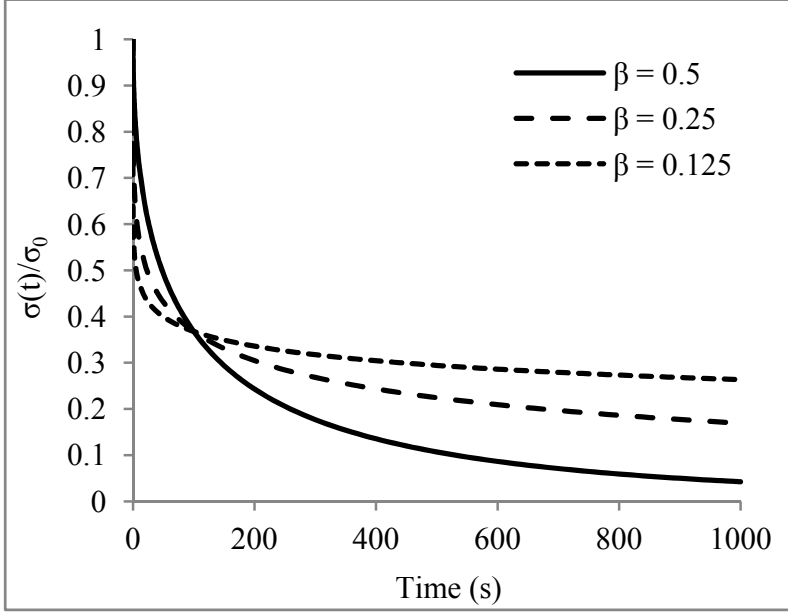

(a)

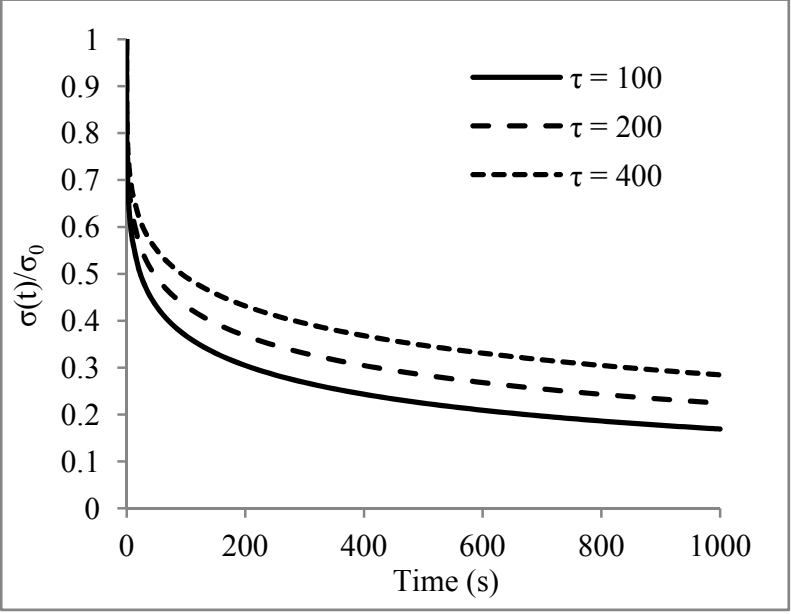

(b)

Fig. 11 Theoretical relaxation curves for different $\beta$ values with $\tau=100$ (a) and different $\tau$ values with $\beta=0.25$ (b). In (a) all the curves intersect at time $\tau$ before separating with the shallower curves corresponding to a lower $\beta$ value. In (b) all the curves maintain a roughly similar shape despite different initial relaxation rates.

of the W-W equation itself [12-15]. As the W-W equation is used as a best fit to the actual data, the calculated values of the equation fitted to the data will follow trends that relate to the nature of the equation.

Since $\tau$ provides no real description of the distribution of relaxation times despite being referred to as a characteristic relaxation time, it is important to consider both $\tau$ and $\beta$ when describing stress relaxation. Many other authors have simplified their analysis of stress relaxation behavior by assuming a constant value for $\beta$ across all the sample materials $[17,18]$. As asserted in this paper, $\beta$ relates to the degree of molecular interaction, which is heavily influenced by water absorption.

\subsection{Adhesive Characteristics}

The adhesive strength of dry and saturated samples is shown in Fig. 13 and Table 4. The adhesive testing 


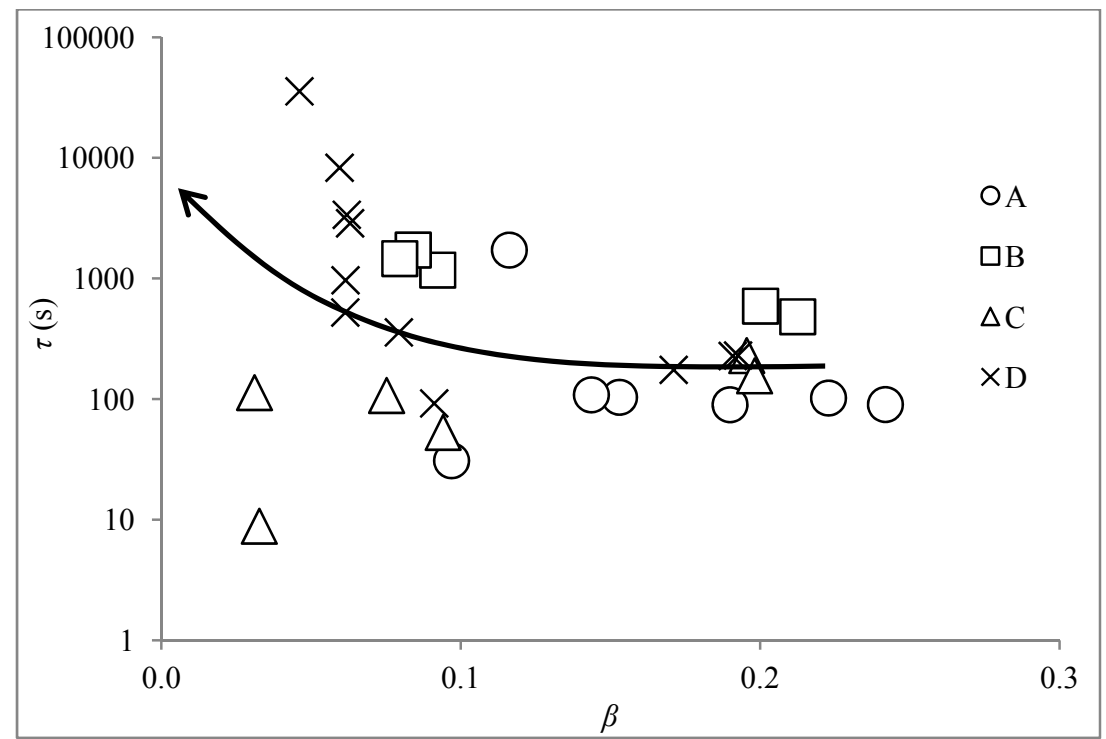

Fig. 12 Correlation of $\tau$ to $\beta$.

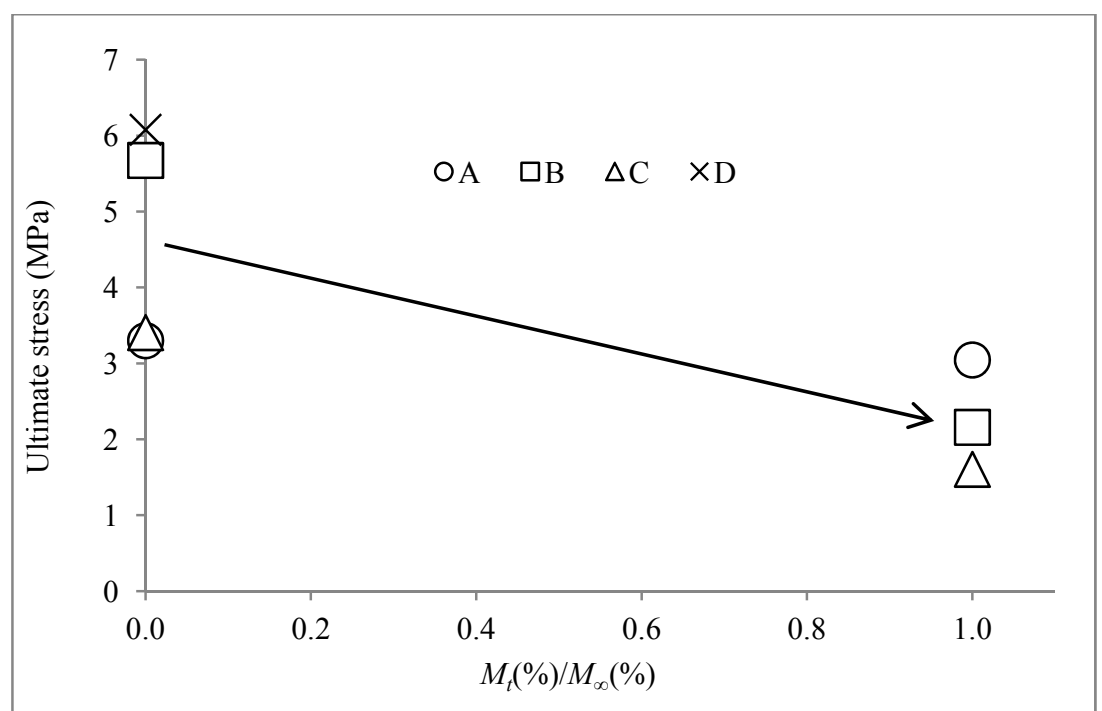

Fig. 13 Adhesive characteristics for dry and saturated samples.

Table 4 Summary of adhesive characteristics for dry and saturated samples.

\begin{tabular}{lllll}
\hline & \multicolumn{3}{c}{ Dry $\left(M_{t} / M_{\infty}=0\right)$} & Saturated $\left(M_{t} / M_{\infty} \approx 1\right)$ \\
\cline { 2 - 5 } & Stress $(\mathrm{MPa})$ & Location of failure & Stress $(\mathrm{MPa})$ & Location of failure \\
\hline $\mathrm{A}$ & $3.30 \pm 1.2$ & $\mathrm{M}$ & $3.05 \pm 0.7$ & $\mathrm{M}$ \\
$\mathrm{B}$ & $5.68 \pm 1.2$ & $\mathrm{M}$ & $2.16^{*}$ & $\mathrm{M} \& \mathrm{E}$ \\
$\mathrm{C}$ & $3.41 \pm 1.3$ & $\mathrm{M}$ & $1.61 \pm 0.5$ & $\mathrm{M}$ \\
$\mathrm{D}$ & $6.08 \pm 0.2$ & $\mathrm{~T}$ & $0.93 \pm 0.4^{* *}$ & $\mathrm{E} / \mathrm{T} \& \mathrm{E}$ \\
\hline
\end{tabular}

Stress indicates ultimate tensile stress. The primary failure location is denoted by an abbreviation for each possible location: "M" bottom or middle coating epoxy layer, "T"- top layer, "E"-adhesive epoxy used to attach testing dolly. Two letters separated by "/" denote the interface between those two layers.

* No error calculated as there was only one successful sample test.

** Failure for coating $\mathrm{D}$ at $M_{t} / M_{\infty} \approx 1$ occurred in the epoxy binding the dolly to the sample, and is thus not indicative of the coating itself. The value listed indicates the stress at which the epoxy between the dolly and the sample failed. The strength of the coating is at least higher than this value. 
of the coatings under dry and saturated conditions indicates that absorbed water has a highly detrimental effect on adhesive strength. Coatings $\mathrm{A}, \mathrm{B}$, and $\mathrm{C}$ showed reductions in adhesive strength of $7 \%, 61 \%$, and $52 \%$, respectively, with the majority cohesive failure where the primary failure plane of all the coatings occur within the bottom or middle coating epoxy layer.

As previously asserted, absorbed water interferes with the intermolecular interactions of the polymer. Cross-linking, in addition to narrowing the distribution of relaxation times, may also narrow the distribution of elastic moduli, and assist if diffusing stress concentrations. As described in Eq. (5), each polymer chain, or mode of the system, has its own elastic modulus, which likely follows a distribution similar to the distribution of $\tau$.

Stress applied to the system will concentrate the largest force in the mode with the highest elastic modulus. Molecular interactions between polymer chains in the system likely help to diffuse this stress concentration. In a saturated system, where the interactions are interrupted, an increase in stress concentration would cause areas of high stress to fail, with the failure propagating through the polymer along a plane perpendicular to the applied stress.

\section{Conclusions}

We have shown that water absorbed into a polymer coating system has a large effect on its material properties. Bonding between water and polymer networks, and the interruption of intermolecular interactions between polymer chains are suggested as major factors contributing to all the results presented in this paper.

The water affinity of coatings has been shown to have a strong positive correlation with $M_{\infty}(\%)$ and $D$ during absorption. These results reinforce the conclusion that a high water affinity supports a higher saturated water content, and helps to facilitate absorption.

Although $\tau$ provides no real description of the distribution of relaxation times, it is indicative of the nature of the relaxation if considered with both $\tau$ and $\beta$. For the three parameters determined in this work, increasing water content was shown to correlate strongly with a reduction in $E_{o}$ and $\beta$ during absorption between $M_{t} / M_{\infty}=0$ and $M_{t} / M_{\infty} \approx 0.5$. This is suggested to be caused by water absorbed early during the absorption process that has a stronger interaction with the polymer network than water absorbed later, and this is corroborated by supporting evidence from other authors and our group.

Finally, the adhesive characteristics of the coatings are correlated with the water content, and shown to exhibit higher cohesive failure in the coating epoxies under saturated conditions. This suggests that water interferes with intermolecular bonding, reduces the bulk materials ability to diffuse stress concentrations and lowers its overall strength.

\section{Acknowledgments}

We thank Mr. R. Nishio of NTT-AT for his assistance with the preparation of coating samples. Most of the content of this paper was mentioned at the technical symposium of STG 33, Chair: Dwight Janoff and Vice Chair: Elizabeth Whitsitt, at NACE CORROSION 2014 in San Antonio in March 2014 and at EMN meeting on polymer, Chair: Arup Neogi and Zhiming Wang, in Orland in Jan 2015 as an invited speech.

\section{References}

[1] Sørensen, P. A., Kiil, S., Dam-Johansen, K., and Weinell, C. E. 2009. "Anticorrosive Coatings: a Review." J. Coat. Technol. Res. 6 (2): 135-176.

[2] Takeshita, Y. 2010. Report on Weathering Technology Acheivements. Tokyo: JWTC, 25-34.

[3] Takeshita, Y., Sakata, S., Sawada, T., Jackson Jr, R. A., and Nishio, R. 2011. "Rheological Property and Adhesive Performance of Polymer Coating Film for Telecommunication Plant." Zairyo-to-Kankyo 60 (3): 147-153.

[4] Takeshita, Y., Jackson Jr, R. A., Sakata, S., Sawada, T., and Nishio, R. 2012. "Aging Property of Eco-Frinedly Anticorrosion Organic Coating Film for Telecommunication Plant." Polym. Eng. Sci. 52 (3): 
572-580. DOI 10.1002/pen.22119.

[5] Kamisho, T., Takeshita, Y., Sakata, S., and Sawada, T. 2013. "Aging Properties of Water-Based and Solvent-Type Coatings under Dry/Wet Accerelated Conditions." Rust Prevention \& Control Japan 57 (12): 451-457.

[6] Kamisho, T., Takeshita, Y., Sakata, S., and Sawada, T. 2014. "Water Absorption of Water-Based Anticorrosive Coating and Its Effect on Mechanical Property and Adhesive Performance." J. Coat. Technol. Res.11 (2): 199-205. DOI 10.1007/s11998-013-9517-z.

[7] Williams, G., and Watts, D. C. 1970. "Non-Symmetrical Dielectric Relaxation Behaviour Arising from a Simple Empirical Decay Function." Transactions of the Faraday Society66: 80-85.

[8] Crank, J. 1975. The Mathematics of Diffusion. Oxford : University Press.

[9] Barton, J. M. 1979. "Effect of Absorbed Water on the Thermal Relaxation of Biaxially Stretched Crosslinked Poly(Methyl Methacrylate)." Polymer 20 (8): 1018-1024.

[10] Nogueira, P., Ramarez, C., Torres, A., Abad, M. J., Cano, J., and Lopez, J. 2001. "Effect of Water Sorption on the Structure and Mechanical Properties of an Epoxy Resin System." Journal of Applied Polymer Science 80 (1): 71-80.

[11] Ferry,J. D. 1980.Viscoelastic Properties of Polymers. New York: John Wiley \& Sons.

[12] Hetman, P., Szabat, B., Weron, K., and Wodziński, D. 2003. "On the Rajagopal Relaxation-Time Distribution and Its Relationship to the Kohlrausch-Williams-Watts Relaxation Function." Journal of Non-Crystalline Solids 330 (1-3): 66-74.

[13] Johnston,D. 2006."Stretched Exponential Relaxation Arising from a Continuous Sum of Exponential Decays." Physical Review B74 (18): 184430-1 184430-7.

[14] Lindsey, C. P., and Patterson, G. D. 1980."Detailed Comparison of the Williams-Watts and Cole-Davidson Functions." The Journal of Chemical Physics 73 (7): 3348-3357.

[15] Weron, K. 1986. "Relaxation in Glassy Materials from Lévy StableDistributions." Acta Physica Polonica. A 70 (5): 529-539.

[16] Smith, T. L. 1979. "Evaluation of the Relaxation Modules from the Response to a Constant Rate of Strain followed by a Constant Strain." Journal of Polymer Science: Polymer Physics Edition 17 (12):2181-2188.

[17] Mijovic, J., Devine, S. T. and Ho, T. 1990. "Physical Aging in Poly (Methyl Methacrylate)/Poly (Styrene-co-Acrylonitrile) Blends. I. Stress Relaxation." 1999. Journal of Applied Polymer Science 39 (5): 1133-1151.

[18] Ho, T., Mijović, J., and Lee, C. 1991. "Effect of Structure on Stress Relaxation of Polymer Blends in Glassy State.' Polymer 32 (4): 619-627.

[19] Rubber. 2005.

Vulcanized or Thermoplastic-Determination of Tensile Stress-Strain Properties. ISO 37:2005.

[20] Soles, C. L., Chang, F. T., Gidley, D. W., and Yee, A. F. 2000. "Contributions of the Nanovoid Structure to the Kinetics of Moisture Transport in Epoxy Resins." $J$. Polym Sci: B, Polym. Phys.38: 776-791.

[21] Baschek, G., Hartwig, G., and Zahradnik, F. 1999. "Effect of Water Absorption in Polymers at Low and High Temperatures." Polymer 40 (12): 3433-3441.

[22] Ping, Z. H., Nguyen, Q. T., Chen, S. M., Zhou, J. Q., and Ding, Y. D. 2001. "States of Water in Different Hydrophilic Polymers-DSC and FTIR Studies." Polymer 42 (20): 8461-8467.

[23] Rosa, F., Bordado, J., and Casquilho, M. 2002. "Kinetics of Water Absorbency in AA/AMPS Copolymers: Applications of a Diffusion-Relaxation Model." Polymer 43 (1): 63-70.

[24] Diamant, Y., Marom, G., and Broutman, L. J. 1981. "The Effect of Network Structure on Moisture Absorption of Epoxy Resins." Journal of Applied Polymer Science 26 (9): 3015-3025.

[25] Hare, C. H. 2001. Paint Film Degradation: Mechanisms and Control. Pittsburgh, SSPC.

[26] Yoon, S. C., and Ratner, B. D. 1988. "Surface and Bulk Structure of Segmented Poly (Ether Urethanes) with Perfluoro Chain Extenders. 2. FTIR, DSC and X-Ray Photoelectron Spectroscopic Studies." Macromolecules 21: 2392-2400

[27] Funke, W., Zorll, U., and Murthy, B. G. 1969. "Interfacial Effects in Solid Paint Films Related to Some Film Properties." Journal Paint Technology 41 (530): 210-221.

[28] Sperling, L. H. 2005. Introduction to Physical Polymer Science.

[29] Bahar, I., Erman, B., Fytas, G., and Steffen, W. 1994. "Intramolecular Contributions to Stretched-Exponential Relaxation Behavior in Polymers." Macromolecules 27 (18): 5200-5205.

[30] Higuchi, A., and Iijima, T. 1985. "D.s.c. Investigation of the States of Water in Poly (Vinyl Alcohol) Membranes." Polymer 26 (8): 1207-1211.

[31] Higuchi, A. and Iijima, T. 1985. "D.s.c. Investigation of the States of Water in Poly (Vinyl Alcohol-co-Itaconic Acid) Membranes." Polymer 26(12): 1833-1837.

[32] Takeshita, Y., Becker, E., Sakata, S., Miwa, T., and Sawada, T. 2014. "States of Water Absorbed in Water-Borne Urethane/Epoxy Coatings." Polymer 55 (10): 2505-2513. 